\title{
A 47-year-old misunderstanding: Indomethacin Polymorph $\delta$ revealed to be two plastically bendable crystal forms by 3D electron diffraction
}

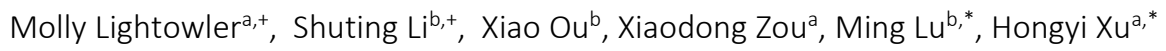 \\ a Department of Materials and Environmental Chemistry, Stockholm University, Stockholm SE-106 91, Sweden \\ b School of Pharmaceutical Sciences, Sun Yat-sen University, Guangzhou 510275, China \\ + : These authors contributed equally. \\ *: Corresponding authors (luming3@mail.sysu.edu.cn, hongyi.xu@mmk.su.se)
}

Keywords: electron diffraction, structure determination, melt crystallization, plasticity, indomethacin, polymorphism

Indomethacin is a clinically classical non-steroidal anti-inflammatory drug that has been marketed since 1965 . The third polymorph, Form $\delta$, was discovered by both melt and solution crystallization in 1974 . $\delta$-indomethacin cannot be cultivated as a large single crystal suitable for $X$-ray crystallography and, therefore, its crystal structure has not yet been determined. Here, we report the structure elucidation of $\delta$-indomethacin by $3 \mathrm{D}$ electron diffraction and reveal the truth that melt-crystallized and solution-crystallized $\delta$-indomethacin are in fact two polymorphs with different crystal structures. Intriguingly, both structures display plastic flexibility based on a slippage mechanism, making indomethacin the first drug to have two plastic polymorphs. This discovery and correction of a 47-year-old misunderstanding signify that 3D electron diffraction has become a powerful tool for polymorphic structural studies.

\begin{abstract}
Polymorphism refers to the formation of different crystal packing from a single compound and is an important phenomenon in many areas of science-most notably the pharmaceutical industry. ${ }^{[1,2]}$ Polymorph screening is a necessary step during drug discovery since different polymorphs of the same drug can differ in their physicochemical properties and drug outcomes. Although solution crystallization is the traditional method for polymorph screening, melt crystallization is now revealing a growing list of pharmaceutical polymorphs which cannot be obtained from solution. ${ }^{[3-}$

${ }^{11]}$ The golden standard for identifying polymorphism is by determining the single crystal structure. Melt crystallization, however, usually yields polycrystalline spherulites and therefore, it is challenging to elucidate the structures of melt-crystallized polymorphs. Recently, we developed a general method for rapidly growing single crystals from melt microdroplets. ${ }^{[12]}$ This strategy highly facilitates the structure determination of polymorphs crystallized from the melt. ${ }^{[3,13]}$
\end{abstract}

Single-crystal X-ray diffraction (SCXRD), the main workhorse in structural chemistry, requires good-sized crystals (usually larger than $50 \mu \mathrm{m}$ in each dimension for laboratory diffractometers and $5-10 \mu \mathrm{m}$ in each dimension for strong synchrotron radiation ${ }^{[14]}$ ) of sufficient quality. Many compounds cannot be grown as large crystals and their small crystals often appear to be poor in quality due to a large mosaic spread and/or stacking faults. ${ }^{[15]}$ Therefore, the structure determination of small crystals (very thin plates or very fine needles) is a difficult and long-standing problem.

3D electron diffraction (3D ED) methods, also known as microcrystal electron diffraction (MicroED), have developed rapidly in recent years. ${ }^{\text {[16-20] }}$ The ability to solve small molecule structures by $3 D$ ED was first explored using test compounds with known structures (organometallic species ${ }^{[21]}$ and pharmaceutical compounds ${ }^{[14,22-24]}$ ) and then compounds with unknown structures (sofosbuvir-L-proline cocrystal, ${ }^{[25]}$ orthocetamol, ${ }^{[26]}$ zeolite $^{2}$ ECNU-23, ${ }^{[27]}$ and loratadine form II[28]). Whilst conceptually 3D ED is comparable to SCXRD, there is a unique advantage to using electrons for diffraction experiments. Thanks to the strong interaction between electrons and matter, 3D electron diffraction data can be collected from very small crystallites. Crystals $10^{6}$ times smaller in volume than those required for X-ray diffraction can now be studied. The power of 3D ED for solving crystal structures of thin crystals has been demonstrated using paracetamol and a methylene blue derivative as model compounds. ${ }^{[14]}$ However, good-quality, large crystals of these two compounds can also be grown meaning the crystals used in the study were also of high-quality despite their small sizes.

Indomethacin (IDM, chemical structure shown in Figure 1) is a clinically classic non-steroidal anti-inflammatory drug discovered in $1963^{[29]}$ and marketed since 1965. This drug displays rich polymorphism. In $1968,{ }^{[30]}$ Yamamoto reported three polymorphs of IDM, $\alpha\left(T_{\mathrm{m}}\right.$ of $\left.154-155.5^{\circ} \mathrm{C}\right), \beta$ and $\gamma\left(T_{\mathrm{m}}\right.$ of $\left.160-161.5^{\circ} \mathrm{C}\right)$, where $T_{\mathrm{m}}$ refers to the melting point. Form $\beta$ was later confirmed to be a solvate of IDM by Joshi et al. in $1998 .{ }^{[31]} \mathrm{In}$ $1974,{ }^{[32]}$ Borka reported the third true polymorph of IDM with a $T_{\mathrm{m}}$ of $134^{\circ} \mathrm{C}$, designated as Form III. Borka grew Form III by two methods: spontaneous crystallization from melt between $70-90^{\circ} \mathrm{C}$ and from a warm methanol solution. In 1998, Joshi obtained Form III by desolvation of IDM solvates ${ }^{[31]}$ and was the first to report the powder X-ray diffraction (PXRD) data. In 2002, Crowley et al. ${ }^{[33]}$ used Joshi's method to prepare Form III by desolvation of IDM methanolate and renamed this polymorph as Form $\delta$. From this point onwards, people referred to the third polymorph (Form III) of IMD obtained by melt crystallization ${ }^{[34,35]}$ and solution crystallization ${ }^{[36]}$ as Form $\delta$. In 2013 , Forms $\varepsilon$, $\eta$ and $\zeta$ were obtained by recrystallization of amorphous IDM in solution under different $\mathrm{pH}$ values. ${ }^{[36]}$ The seventh polymorph was discovered in 2018 by recrystallization of IDM from polyethylene glycol-based solid dispersion..$^{[37]}$ Only the crystal structures of Forms $\alpha(P \overline{1}, Z=2 \text {, Table S1) })^{[38]}$ and $\beta\left(P 2_{1}, Z=6, \text { Table S1 }\right)^{[39]}$ are known and were reported in 1972 and 2002, respectively. 


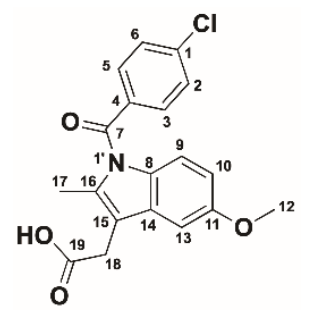

Figure 1. The chemical structure of indomethacin.

We noticed that the reported PXRD patterns of $\delta$-IDM crystallized from melt ${ }^{[3,35]}$ and solution ${ }^{[31,33,36]}$ are different. This led us to ask, do the two crystallization methods of $\delta$-IDM produce different polymorphs? In this work, we performed microdroplet melt crystallization ${ }^{[12]}$ for single crystal cultivation of both melt-crystallized $\delta$-IDM (melt $\delta$-IDM) and solution-crystallized $\delta$-IDM (solution $\delta$-IDM ) and applied 3D ED for structure elucidation of both phases to correct this 47-year-old misunderstanding.

We grew polycrystalline melt $\delta$-IDM by spontaneous nucleation at $60^{\circ} \mathrm{C}$ from supercooled IDM. We obtained polycrystalline solution $\delta$-IDM from a pH 6.8 phosphate-buffered saline by partial recrystallization of amorphous IDM, then seeded it in supercooled IDM at $100{ }^{\circ} \mathrm{C}$ for growth. The PXRD patterns of melt and solution $\delta$-IDM matched the reported data well (Figure 2a and S1). ${ }^{[31,33-36]}$ Further characterization indicated that melt and solution $\delta$-IDM samples exhibited different melting enthalpies $(26.53 \pm 0.69 \mathrm{~J} / \mathrm{mol}$ for melt $\delta$-IDM and $30.78 \pm 0.38 \mathrm{~J} / \mathrm{mol}$ for solution $\delta$-IDM, $n=3$ ) and Fourier transform Raman and infrared spectra (Figures 2b-d, S2 and S3). This confirmed our hypothesis that the two methods of crystallization produce different polymorphs. The two phases have very similar melting points $(134.17 \pm 0.76$ for melt $\delta$-IDM and $133.57 \pm 0.29$ for solution $\delta$-IDM, peak temperature, $n=3$, Table S2), which may be the reason why they have been mistaken for the same polymorph for almost five decades.
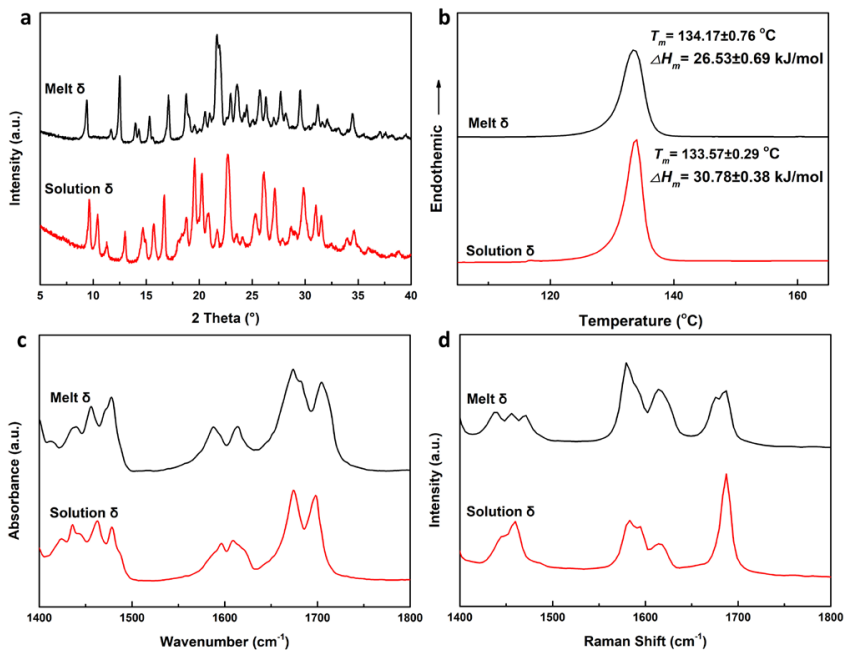

Figure 2. Characterization of melt $\delta$-IDM and solution $\delta$-IDM. (a) PXRD patterns; (b) Differential scanning calorimetry (DSC) curves ( $n=3$ ); (c) Fourier transform infrared spectra; (d) Fourier transform Raman spectra

To unambiguously confirm the two phases, we needed to determine their single crystal structures. Single crystals of melt and solution $\delta$-IDM were cultivated from supercooled IDM by microdroplet melt crystallization. ${ }^{[12]}$ Single crystals of melt $\delta$-IDM are ribbons (Figures $3 a$ and S4) and those of solution $\delta$-IDM are needles (Figures $3 \mathrm{~d}$ and S4). Both crystals exhibit one-dimensional growth and are too thin for sufficient X-ray crystallographyeven the third-generation synchrotron radiation source. The small size of the single crystals may be the reason why the two structures of $\delta$-IDM have remained unknown for such a long time. 

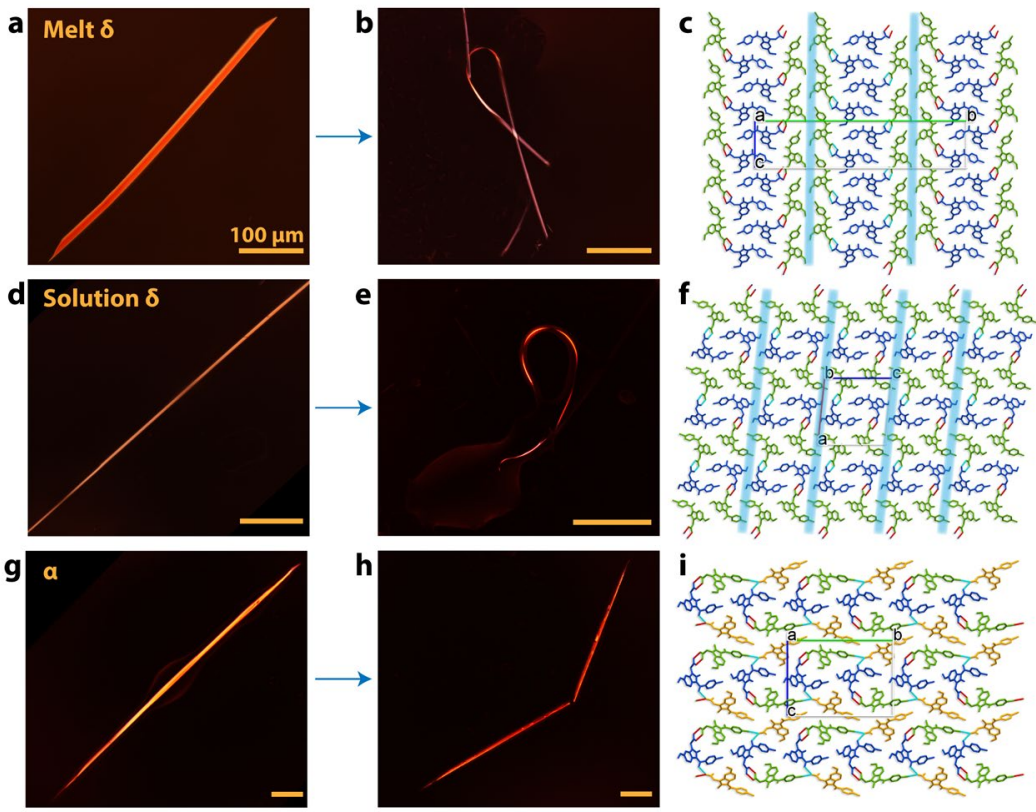

Figure 3. Polarized optical microscopy images of single crystals and crystal structures of IDM polymorphs. (a-c) Melt $\delta$-IDM; (d-f) Solution $\delta$-IDM; (g-i) $\alpha$-IDM. Left: single crystal before deformation. Middle: single crystal after deformation. Right: crystal packing.

For 3D ED data collection, we first separated the single crystals from the supercooled melt, gently crushed them and then loaded them on a holey carbon film supported Cu TEM grid. Data were collected from both melt and solution $\delta$-IDM at room temperature using the continuous rotation method. ${ }^{[16-19,40]}$ Since the samples were beam sensitive, data were collected in small wedges $\left(\sim 40^{\circ}\right)$ over a large tilt range $\left(-60^{\circ}\right.$ to $\left.+60^{\circ}\right)$ which helped to achieve sufficient completeness whilst avoiding significant radiation damage. The crushed crystals had a width typically of $1-2.5 \mu \mathrm{m}$ (Figure S5) and diffracted to a resolution of $\sim 1 \AA$ for melt $\delta$-IDM and $1.15 \AA$ for solution $\delta$-IDM. The diffraction frames were indexed using Rotation ED processing software $(R E D p)^{[41]}$ and integrated and merged using XDS. ${ }^{[42]}$ The unit cell parameters were refined against the PXRD data using the Pawley method ${ }^{[33]}$ with the TOPAS-Academic v6 program package. ${ }^{[44]}$

Melt $\delta$-IDM is in the C-centered monoclinic space group CC (No. 9) with the lattice parameters: $a=4.786(1) \AA, b=56.999(9) \AA, c=12.908(2) \AA$, $\alpha=$ $90^{\circ}, B=99.57(1)^{\circ}, v=90^{\circ}$ (Figures 4 and S6). Since diffraction data were collected in small wedges, it was necessary to merge individual datasets after indexing to improve completeness and $I / \sigma(I)$ for structure solution and refinement. Seven datasets were chosen for merging based on the similarity of their unit cell dimensions and cross-correlation of indexed intensities, giving an overall completeness of 74.5\% (Table S3 summarizes the crystallographic information used for the structure solution). Although the merged data had sufficient resolution ( $1.02 \AA$ ), completeness (74.5 $\%)$ and $I / \sigma(I)(3.7)$, the structure could not be solved by direct methods. This was due to missing diffraction information along the $0 \mathrm{k} 0$ axis (Figures 4 and S6). The preferred orientation of the crystals on the grid together with the limited rotation range of the specimen holder prevented the collection of diffraction information along the $0 \mathrm{KO}$ axis. We overcame the problem of missing information by utilizing the simulated annealing method implemented in the program Sir2014 ${ }^{[45]}$ as the method for phasing. Woollam et al. ${ }^{[28]}$ demonstrated recently that simulated annealing is a convenient alternative method to reach a structure solution when 3D ED data are not sufficient for structure solution by ab initio methods. The simulated annealing input requires a molecular model along with the merged ED data. The atom connectivity of $\gamma$-indomethacin (as reported in the CSD, reference code INDMET ${ }^{[38]}$ ) was used to create the rigid-body starting fragment in the form of a mol file. According to volume calculations, the asymmetric unit was predicted to contain two crystallographically independent molecules, therefore, two starting fragments were used as the input together with the experimental data. The general conditions for simulated annealing can be found in Table S4. Preliminary structure solutions showed overlapping molecules, this was again due to the lack of diffraction data along the 0k0 axis. Anti-bumping restraints were then used to prevent the overlapping. The structure with the lowest cost function ( CF $=23.67)$ was refined by least-squares against the ED data using SHELXL. ${ }^{[46]}$ The final R factor (R1 for all reflections) was 24.9\%; the structure is shown in Figure 5 (Table S5 summarizes the experimental crystallographic and refinement data). 

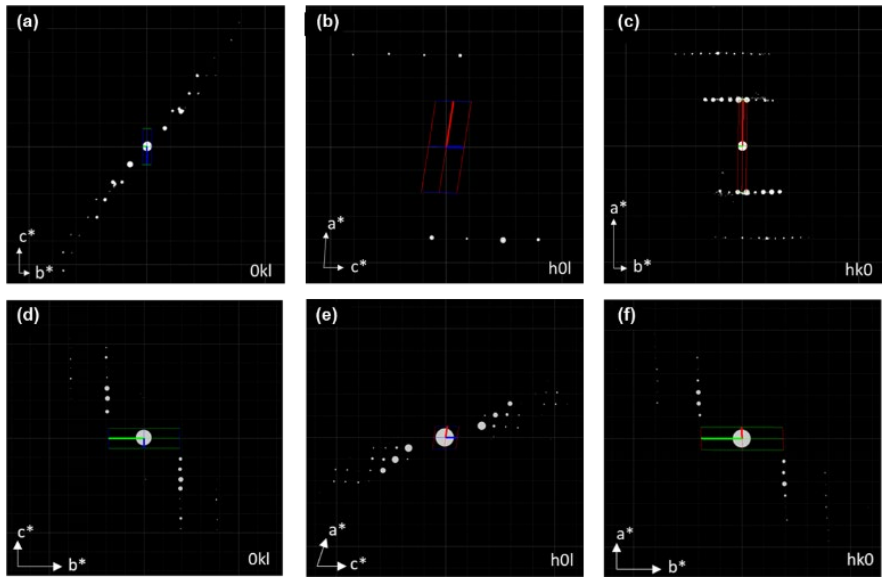

Figure 4. 2D slices of reciprocal lattice planes. (a - c) melt $\delta$-IDM; The reflection conditions obtained from the 3D ED data show that the crystal space group is Cc (No. 9). ( $d$ - $f$ ) solution $\delta$-IDM; The reflection conditions show that the crystal space group is $P 2_{1}$ (No. 4).

Solution $\delta$-IDM is in the monoclinic space group $P 2_{1}$ (No.4) with the unit cell parameters: $a=18.301(5) \AA, b=5.123(1) \AA, c=18.564(6) \AA, \alpha=90^{\circ}$, $B=95.80(1)^{\circ}, y=90^{\circ}$ (Figures 4 and S7). Five datasets were chosen for merging and the overall completeness was $75.0 \%$ (Table S6 summarizes the crystallographic information used for the structure solution). The structure was solved using the simulated annealing method implemented in the program Sir2014, ${ }^{[45]}$ using the same general conditions as melt $\delta$-IDM (Table S4). The structure with the lowest cost function $(C F=29.20)$ was refined by least-squares against the ED data using SHELXL. ${ }^{[46]}$ The final $R$ factor was 19.8\%; the structure is shown in Figure 6 (Table S7 summarizes the experimental crystallographic and refinement data). The simulated and experimental PXRD patterns for both melt and solution $\delta$-IDM correlate well (Figures S8 and S9), confirming the correct structure elucidation and homogeneity for both phases.

The structure of melt $\delta$-IDM exhibited an extremely long $b$-axis (12 times the $a$-axis and 4.5 times the $c$-axis). By comparing the crystal orientations in real-space images with the corresponding indexed diffraction patterns (Figures S10 and S11), the longest crystal dimension was determined to be along the $a$-axis while the shortest dimension is along the $b$-axis This phenomenon matches the well-observed rule that the fastest growth of crystal occurs along the shortest crystallographic axis. ${ }^{[47,48]}$ The huge difference in size between the $b$-axis and the other axes may be one of the essential reasons for the one-dimensional growth and resulting thin morphology of melt $\delta$-IDM. However, solution $\delta$-IDM has a smaller difference in the size of the three crystal axes but a much finer morphology in comparison to melt $\delta$-IDM. 


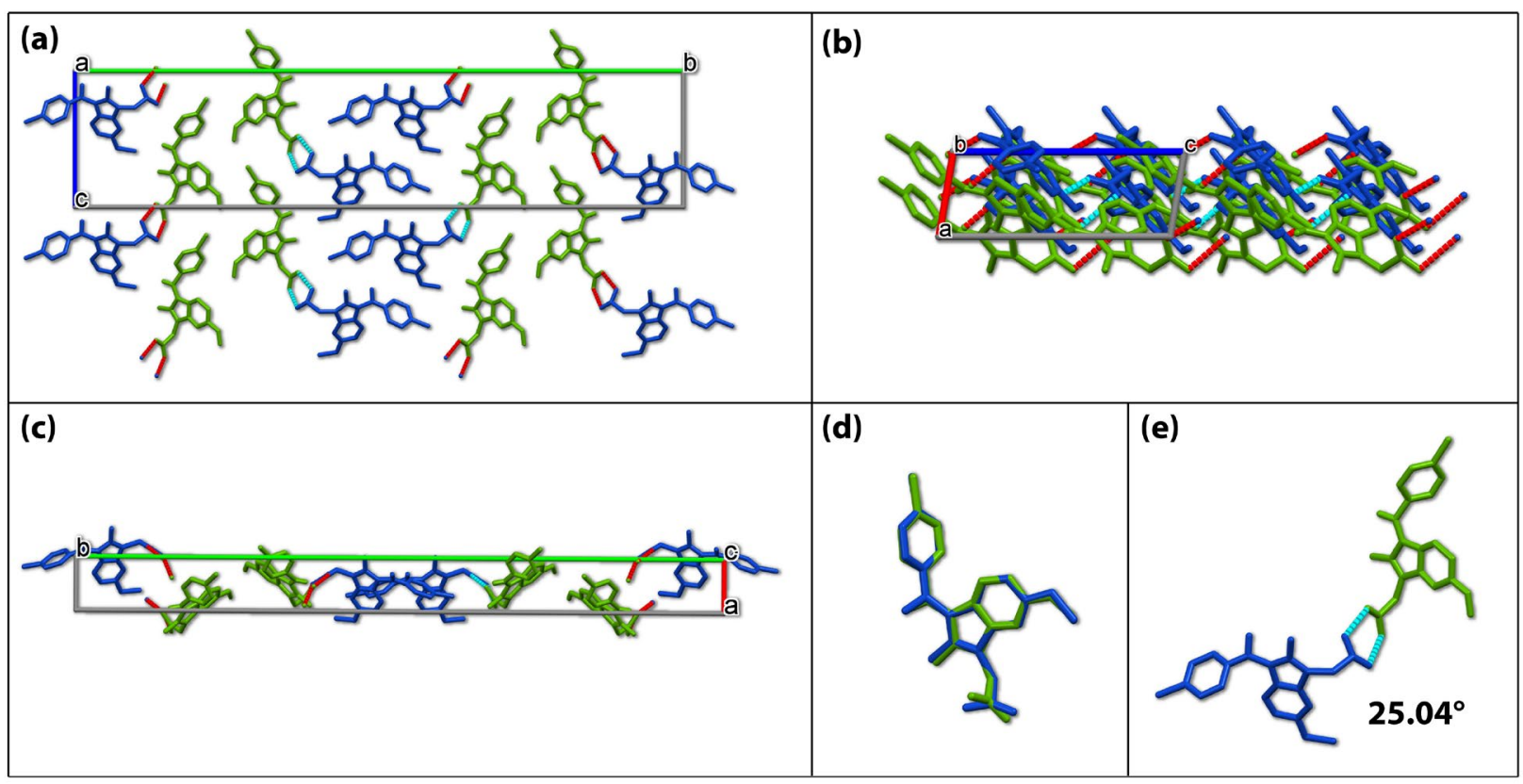

Figure 5. Crystal structure of melt $\delta$-IDM. (a) Molecular packing in melt $\delta$-IDM viewed along the a-axis. (b) Viewed along the b-axis. (c) Viewed along the c-axis. (d) Overlay of the two crystallographically independent molecules (blue: molecule 1; green: molecule 2). (e) Carboxylic acid dimer formed between the two crystallographically independent molecules with a dihedral angle of $25.04^{\circ}$.

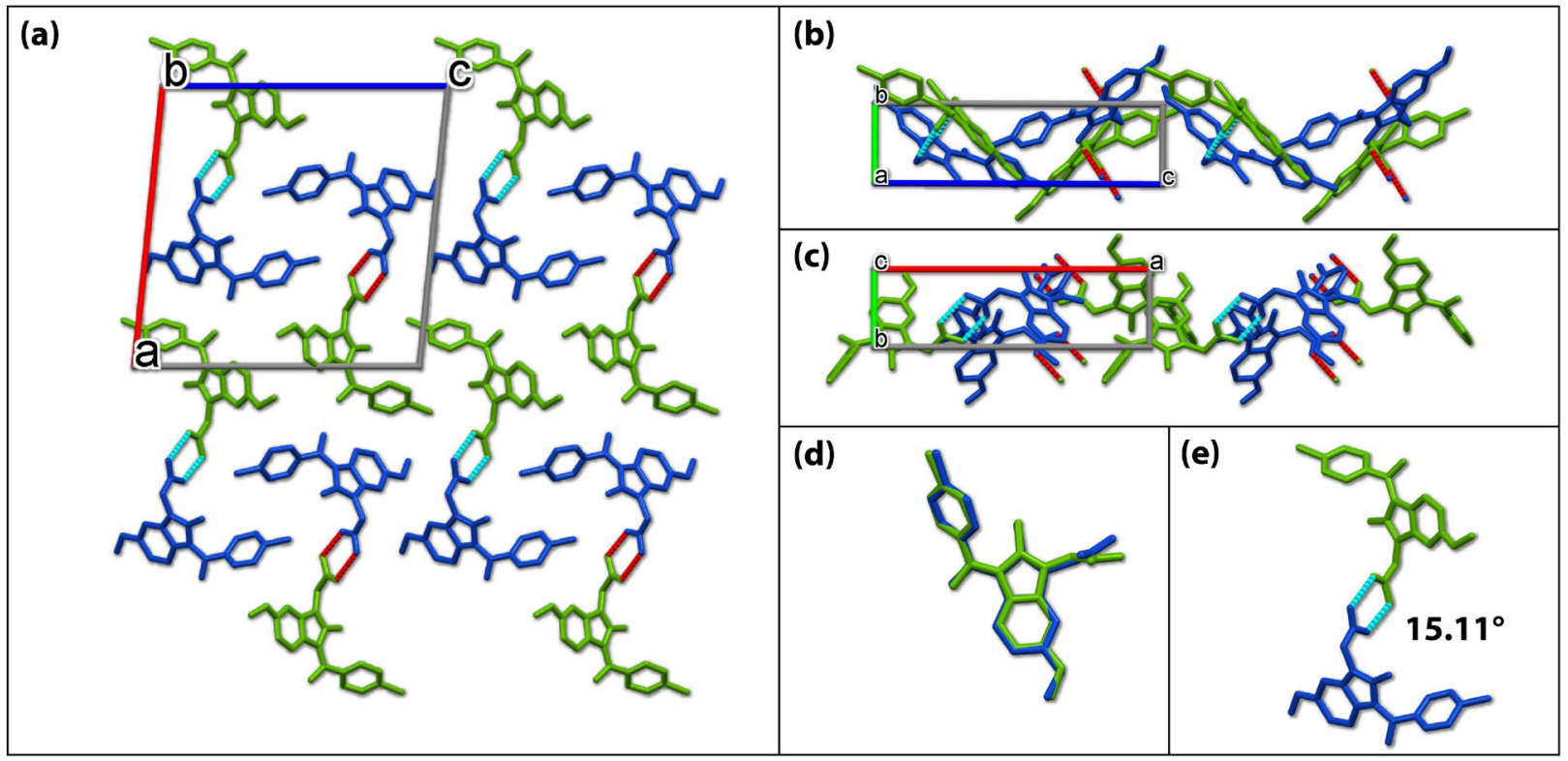

Figure 6. Crystal structure of solution S-IDM. (a) Molecular packing in solution $\delta$-IDM viewed along the b-axis. (b) Viewed along the a-axis. (c) Viewed along the c-axis. (d) Overlay of the two crystallographically independent molecules (blue: molecule 1; green: molecule 2). (e) Carboxylic acid dimer formed between the two crystallographically independent molecules with a dihedral angle of $15.11^{\circ}$. 
For both melt and solution $\delta$-IDM, the asymmetric unit contained two crystallographically independent molecules with very similar conformations, between which a carboxylic acid dimer forms (Figures 5 e and $6 e$ ). This dimer also exists in $\alpha$ - and $\psi$-IDM. The dihedral angle in melt $\delta$-IDM is $25.04^{\circ}$, higher than that in solution $\delta$-IDM $\left(15.11^{\circ}\right), \alpha-\operatorname{IDM}\left(11.76^{\circ}\right)^{[39]}$ and $\gamma$-IDM $\left(0^{\circ}\right)^{[38]}$. As shown in Table 88 , the eight conformations found in the four IDM polymorphs can be classified into two families based on the torsion angles of $\theta$ (C4-C7-N1'-C16): molecule 1 in $\gamma^{-I D M}\left(151.09^{\circ}\right)$ and molecules 1 and 2 in $\alpha$-IDM $\left(154.54^{\circ}\right.$ and $\left.153.64^{\circ}\right)$ and melt $\delta$-IDM $\left(159.85^{\circ}\right.$ and $\left.152.90^{\circ}\right)$ belong to the same conformational family, while molecule 3 in $\alpha-$ $\operatorname{IDM}\left(22.74^{\circ}\right)$ and molecules 1 and 2 in solution $\delta$-IDM $\left(26.26^{\circ}\right.$ and $\left.27.58^{\circ}\right)$ belong to another group.

Interestingly, both the ribbon-like melt $\delta$-IDM and needle-like solution $\delta$-IDM can undergo one-dimensional plastic deformation in the melt at $120^{\circ} \mathrm{C}$ (Figure $3 \mathbf{c}$ and $\mathbf{f}$ ), while needle-like $\alpha$-IDM is brittle and easy to break under the same conditions (Figure $3 \mathrm{~h}$ ). Since single crystals of $\gamma$-IDM have a block shape, it was not included in this discussion. Melt $\delta$-IDM single crystals are readily bent down the main face (020) (i.e.ac plane, perpendicular to the longest crystal axis, $b$-axis) into the desired shape. After the force is removed, the crystals retain their bent shape. The origianl shape can be almost revovered with further application of external force. However, if the force is applied to the narrow side of the ribbon (along the shortest axis in geometry), it can easily break, indicative of one-dimensional plastic deformation for melt $\delta$-IDM. Solution $\delta$-IDM exhibits similar plasticity. It is difficult to distinguish the main faces (001) and (100) of solution $\delta$-IDM, which are parallel to the shortest crystal axis (b-axis) because these needle-like crystals are extremely fine. In our experiments, some solution $\delta$-IDM single crystals are very brittle and others are plastically bendable. Therefore, we speculate that this difference in mechanical deformation originates from the external force exerted on different crystal faces and plastically deformation of solution $\delta$-IDM is also one-dimensional.

In melt $\delta$-IDM, each crystallographically independent molecule packs along the $a$-axis to form $\pi-\pi$ stacking between the indole and chlorobenzene rings. Each blue molecule forms a carboxylic acid dimer $\left(2.490 \AA\right.$, $\left.2.868 \AA, 25.04^{\circ}\right)$ with the neighboring green molecule and the two molecules are nearly perpendicular. These right-angle dimers interlock along the $c$-axis to form zippers. Different zipper columns are linked by weak van der Waals interactions, which facilitates the slipping between columns under stress. These slip planes are perpendicular to the $b$-axis (the shortest crystal axis) and parallel to the major face (020) (shown in Figures $3 c$ and 7a). In solution $\delta$-IDM, each molecule in the asymmetric unit packs along the $b$ axis, forming $\pi-\pi$ stacking between the indole and chlorobenzene rings. The two crystallographically independent molecules form Z-shaped carboxylic acid dimers $\left(2.859 \AA, 2.684 \AA, 15.11^{\circ}\right)$ with each other, which are arranged in rows along the $a$-axis (shown in Figures $3 f$ and $\left.7 b\right)$. Weak van der Waals interactions between rows contribute to the formation of slip planes parallel to the face (001). Both melt and solution $\delta$-IDM have $\pi-\pi$ stacking columns along the shortest crystal axis ( $a$-axis for melt $\delta$-IDM and $b$-axis for solution $\delta$-IDM) and the slip planes form between molecular columns, while the slip planes cannot be found in brittle $\alpha$-IDM (Figures $7 c, f$ and $i$ ). Therefore, the slippage of molecular layers along the slip plane is proposed to be the mechanism of plastic deformation of melt and solution $\delta$-IDM during bending (see schematic diagram in Figure 6). The layer slippage enables the deformation to occur and the weak interactions between the layers makes the deformation irreversible. This slippage mechanism is the most common mechanism of plastic bending for molecular crystals. ${ }^{[49-54]}$ No slip planes can be observed along the b-axis for melt $\delta$-IDM nor parallel to the (100) plane for solution $\delta$-IDM. This explains the one-dimensional plastic flexibility of the two polymorphs well.
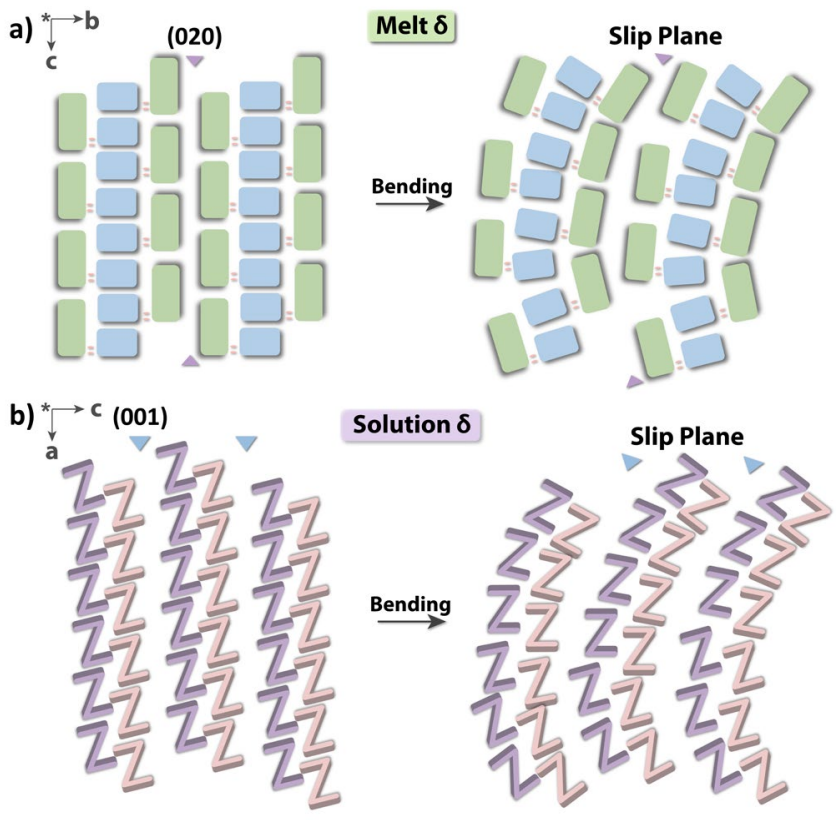

Figure 7. Schematic diagram of bending deformation of IDM polymorphs. (a) Melt $\delta$-IDM; (b) Solution S-IDM. 
Most organic molecular crystals are typically fragile and brittle under excessive stress and plasticity is rare. One compound displaying multiple plastic polymorphs is particularly remarkable. To the best of our knowledge, IDM is the first clinical drug with two phase-pure polymorphs displaying plasticity. Polymorphism that showcases various mechanical behaviors helps us to understand the relationship between crystal structure and solidstate properties ${ }^{[55-58]}$ and also offers an excellent opportunity to tune or predict pharmaceutical-related mechanical properties. ${ }^{[59]}$ For example, the compressibility of an active pharmaceutical ingredient plays an important role in the tabletability of the formulation, especially for high drug-load formulations. ${ }^{[60]}$ Plastic flexibility is advantageous for the tabletability due to the possibility of irreversible plastic deformation via the slippage of molecular layers and resulting improved ability to accommodate stress. ${ }^{[52]}$ Therefore, the tabletability of melt and solution $\delta$-IDM deserves further exploration due to their excellent plasticity compared to other polymorphs, for a better understanding of the relationship between pharmaceuticalrelated mechanical properties and crystal structure and tuning of the tabletability through crystal engineering. Most plastically bendable molecular crystals were reported at room temperature, while only limited systems were studied above or below room temperature. ${ }^{[6] 1}$ Because both melt and solution $\delta$-IDM exhibit plastic flexibility at a high temperature $\left(120^{\circ} \mathrm{C}\right)$, the dependence of mechanical properties of IDM polymorphs on temperature is worthy of further investigation.

Through combining microdroplet melt crystallization for single crystal growth with 3D ED for structure determination, we reported that the wellstudied $\delta$-IDM samples crystallized from melt and solution are two phases with very similar $T_{\mathrm{m}}$ but different crystal structures. For 47 years, it has been understood that the two methods of crystallization produce the same polymorph, this finding now corrects that misunderstanding. Single crystals of both $\delta$-IDM phases are extremely thin, meaning the structures could not be determined by X-ray crystallography-even the thirdgeneration synchrotron radiation. By finally achieving structure determination by $3 D E D$, the first case of a clinical drug displaying two phase-pure polymorphs with plastically bendable properties was revealed and the potential to tune the mechanical properties of drugs by polymorph selection was suggested. The successful single crystal growth and structure elucidation of both phases highlight the advancements in crystallization technology and emphasize the importance of 3D ED in polymorphic structural studies, especially for cases of very thin crystals that are beyond the capability of X-ray crystallography.

\section{Acknowledgements}

We are grateful to A. K. Inge, Stockholm University, for his expertise and support regarding the work relating to X-ray diffraction and to Prof. Bin Tian (Shaanxi University of Science and Technology, China) for his helpful discussion regarding Indomethacin polymorph $\delta$. We acknowledge funding of the Swedish Research Council (2017-05333, 2019-00815), the Knut and Alice Wallenberg Foundation (2018.0237), SciLifeLab technology development project (MicroED@SciLifeLab) and Guangdong Basic and Applied Basic Research Foundation (No. 2020A1515010782).

\section{Bibliography}

[1] A. J. Cruz-Cabeza, S. M. Reutzel-Edens, J. Bernstein, Chem. Soc. Rev. 2015, 44, 8619-8635.

[2] D. K. Bučar, R. W. Lancaster, J. Bernstein, Angew. Chemie - Int. Ed. 2015, 54, 6972-6993.

[3] X. Li, X. Ou, B. Wang, H. Rong, B. Wang, C. Chang, B. Shi, L. Yu, M. Lu, Commun. Chem. 2020, 3, DOI 10.1038/s42004-020-00401-1.

[4] Y. Gui, X. Yao, I. A. Guzei, M. M. Aristov, J. Yu, L. Yu, Chem. Mater. 2020, 32, 7754-7765.

[5] K. Zhang, N. Fellah, A. G. Shtukenberg, X. Fu, C. Hu, M. D. Ward, CrystEngComm 2020, 22, 2705-2708.

[6] D. Skomski, R. J. Varsolona, Y. Su, J. Zhang, R. Teller, S. P. Forster, S. E. Barrett, W. Xu, Mol. Pharm. 2020, 17, $2874-2881$.

[7] A. G. Shtukenberg, M. Tan, L. Vogt-Maranto, E. J. Chan, W. Xu, J. Yang, M. E. Tuckerman, C. T. Hu, B. Kahr, Cryst. Growth Des. 2019, 19, 4070-4080.

[8] M. A. Ciciliati, M. E. S. Eusébio, M. R. Silva, É. T. G. Cavalheiro, R. A. E. Castro, CrystEngComm 2019, 21, 4319-4328.

[9] Q. Zhu, A. G. Shtukenberg, D. J. Carter, T. Q. Yu, J. Yang, M. Chen, P. Raiteri, A. R. Oganov, B. Pokroy, I. Polishchuk, P. J. Bygrave, G. M. Day, A. L. Rohl, M. E. Tuckerman, B. Kahr, J. Am. Chem. Soc. 2016, 138, 4881-4889.

[10] C. Yao, I. A. Guzei, Y. Jin, S. Ruan, G. Sun, Y. Gui, L. Wang, L. Yu, Cryst. Growth Des. 2020, 20, 7874-7881.

[11] N. Fellah, A. G. Shtukenberg, E. J. Chan, L. Vogt-Maranto, W. Xu, C. Li, M. E. Tuckerman, B. Kahr, M. D. Ward, Cryst. Growth Des. 2020, 20, 2670-2682.

[12] X. Ou, X. Li, H. Rong, L. Yu, M. Lu, Chem. Commun. 2020, 56, 9950-9953.

[13] X. Li, X. Ou, H. Rong, S. Huang, J. Nyman, L. Yu, M. Lu, Cryst. Growth Des. 2020, 20, 7093-7097.

[14] T. Gruene, J. T. C. Wennmacher, C. Zaubitzer, J. J. Holstein, J. Heidler, A. Fecteau-Lefebvre, S. De Carlo, E. Müller, K. N. Goldie, I. Regeni, T. Li, G. Santiso-Quinones, G. Steinfeld, S. Handschin, E. van Genderen, J. A. van Bokhoven, G. H. Clever, R. Pantelic, Angew. Chemie Int. Ed. 2018, 57, 16313-16317.

[15] M. M. Harding, IUCr, J. Synchrotron Rad. 1996, 3, 250-259. 
[16] I. Nederlof, E. Van Genderen, Y. W. Li, J. P. Abrahams, Acta Crystallogr. Sect. D Biol. Crystallogr. 2013, 69, 1223-1230.

[17] M. Gemmi, M. G. I. La Placa, A. S. Galanis, E. F. Rauch, S. Nicolopoulos, J. Appl. Crystallogr. 2015, 48, 718-727.

[18] B. L. Nannenga, D. Shi, A. G. W. Leslie, T. Gonen, Nat. Methods 2014, 11, 927-930.

[19] Y. Wang, S. Takki, O. Cheung, H. Xu, W. Wan, L. Öhrström, A. K. Inge, Chem. Commun. 2017, 53, 7018-7021.

[20] M. Gemmi, E. Mugnaioli, T. E. Gorelik, U. Kolb, L. Palatinus, P. Boullay, S. Hovmöller, J. P. Abrahams, ACS Cent. Sci. 2019, 5, 1315-1329.

[21] C. G. Jones, M. Asay, L. J. Kim, J. F. Kleinsasser, A. Saha, T. J. Fulton, K. R. Berkley, D. Cascio, A. G. Malyutin, M. P. Conley, B. M. Stoltz, V. Lavallo, J. A. Rodríguez, H. M. Nelson, ACS Cent. Sci. 2019, 5, 1507-1513.

[22] C. G. Jones, M. W. Martynowycz, J. Hattne, T. J. Fulton, B. M. Stoltz, J. A. Rodriguez, H. M. Nelson, T. Gonen, ACS Cent. Sci. 2018, 4, $1587-1592$.

[23] T. E. Gorelik, J. van de Streek, A. F. M. Kilbinger, G. Brunklaus, U. Kolb, Acta Crystallogr. Sect. B 2012, 68, 171-181.

[24] E. Van Genderen, M. T. B. Clabbers, P. P. Das, A. Stewart, I. Nederlof, K. C. Barentsen, Q. Portillo, N. S. Pannu, S. Nicolopoulos, T. Gruene, J. P. Abrahams, Acta Crystallogr. Sect. A Found. Adv. 2016, 72, 236-242.

[25] P. Brázda, L. Palatinus, M. Babor, Science (80-. ). 2019, 364, 667-669.

[26] I. Andrusenko, V. Hamilton, E. Mugnaioli, A. Lanza, C. Hall, J. Potticary, S. R. Hall, M. Gemmi, Angew. Chemie - Int. Ed. 2019, 58, 1091910922.

[27] X. Liu, Y. Luo, W. Mao, J. Jiang, H. Xu, L. Han, J. Sun, P. Wu, Angew. Chemie - Int. Ed. 2020, 59, 1166-1170.

[28] G. R. Woollam, P. P. Das, E. Mugnaioli, I. Andrusenko, A. S. Galanis, J. Van De Streek, S. Nicolopoulos, M. Gemmi, T. Wagner, CrystEngComm 2020, 22, 7490-7499.

[29] F. D. Hart, P. L. Boardman, Br. Med. J. 1963, 2, 965-970.

[30] H. Yamamoto, Chem. Pharm. Bull. (Tokyo). 1968, 16, 17-19.

[31] Vidya Joshi, Physical Transformations in Solvated Pharmaceuticals, Purdue University, 1998.

[32] L. Borka, Acta Pharm. Suec. 1974, 11, 295-303.

[33] G. Zografi, K. J. Crowley, J. Pharm. Sci. 2002, 91, 492-507.

[34] T. Wu, L. Yu, J. Phys. Chem. B 2006, 110, 15694-15699.

[35] B. Tian, W. Gao, X. Tao, X. Tang, L. S. Taylor, Cryst. Growth Des. 2017, 17, 6467-6476.

[36] S. A. Surwase, J. P. Boetker, D. Saville, B. J. Boyd, K. C. Gordon, L. Peltonen, C. J. Strachan, Mol. Pharm. 2013, 10, 4472-4480.

[37] T. Van Duong, D. Lüdeker, P. J. Van Bockstal, T. De Beer, J. Van Humbeeck, G. Van Den Mooter, Mol. Pharm. 2018, 15, $1037-1051$.

[38] T. J. Kistenmacher, R. E. Marsh, J. Am. Chem. Soc. 1972, 94, 1340-1345.

[39] X. Chen, K. R. Morris, U. J. Griesser, S. R. Byrn, J. G. Stowell, J. Am. Chem. Soc. 2002, 124, 15012-15019.

[40] Y. Wang, T. Yang, H. Xu, X. Zou, W. Wan, J. Appl. Crystallogr. 2018, 51, 1094-1101.

[41] W. Wan, J. Sun, J. Su, S. Hovmöller, X. Zou, J. Appl. Crystallogr. 2013, 46, 1863-1873.

[42] W. Kabsch, Acta Crystallogr. Sect. D Biol. Crystallogr. 2010, 66, 125-132.

[43] G. S. Pawley, J. Appl. Cryst 1981, 14, 357-361.

[44] A. Coelho, 2016.

[45] M. C. Burla, R. Caliandro, B. Carrozzini, G. L. Cascarano, C. Cuocci, C. Giacovazzo, M. Mallamo, A. Mazzone, G. Polidori, J. Appl. Crystallogr. 2015, 48, 306-309.

[46] G. M. Sheldrick, Acta Crystallogr. Sect. C Struct. Chem. 2015, 71, 3-8.

[47] K. V. Rajendran, D. Jayaraman, R. Jayavel, P. Ramasamy, J. Cryst. Growth 2003, 255, 361-368.

[48] S. Manivannan, S. Dhanuskodi, J. Cryst. Growth 2004, 262, 473-478. 
[49] A. Hasija, D. Chopra, CrystEngComm 2021, DOI 10.1039/d1ce00173f.

[50] S. Bhandary, A. J. Thompson, J. C. McMurtrie, J. K. Clegg, P. Ghosh, S. R. N. K. Mangalampalli, S. Takamizawa, D. Chopra, Chem. Commun. 2020, 56, 12841-12844.

[51] A. Mondal, B. Bhattacharya, S. Das, S. Bhunia, R. Chowdhury, S. Dey, C. M. Reddy, Angew. Chemie - Int. Ed. 2020, 59, 10971-10980.

[52] S. Hu, M. K. Mishra, C. C. Sun, Chem. Mater. 2019, 31, 3818-3822.

[53] G. R. Krishna, R. Devarapalli, G. Lal, C. M. Reddy, J. Am. Chem. Soc. 2016, 138, 13561-13567.

[54] S. Saha, M. K. Mishra, C. M. Reddy, G. R. Desiraju, Acc. Chem. Res. 2018, 51, 2957-2967.

[55] K. Zhang, C. C. Sun, Y. Liu, C. Wang, P. Shi, J. Xu, S. Wu, J. Gong, Chem. Mater. 2021, 33, 1053-1060.

[56] M. K. Mishra, C. C. Sun, Cryst. Growth Des. 2020, 20, 4764-4769.

[57] X. Chu, Z. Lu, B. Tang, B. Liu, K. Ye, H. Zhang, J. Phys. Chem. Lett. 2020, 11, 5433-5438.

[58] K. B. Raju, S. Ranjan, V. S. Vishnu, M. Bhattacharya, B. Bhattacharya, A. K. Mukhopadhyay, C. M. Reddy, Cryst. Growth Des. 2018, 18, 3927-3937.

[59] J. A. Yadav, K. S. Khomane, S. R. Modi, B. Ugale, R. N. Yadav, C. M. Nagaraja, N. Kumar, A. K. Bansal, Mol. Pharm. $2017,14,866-874$.

[60] T. V Joshi, A. B. Singaraju, H. S. Shah, K. R. Morris, L. L. Stevens, R. V Haware, Cryst. Growth Des. 2018, 18, 5853-5865.

[61] H. Liu, K. Ye, Z. Zhang, H. Zhang, Angew. Chemie 2019, 131, 19257-19262. 